\title{
Bildung von van der Waals-Komplexen organischer Moleküle
}

\author{
Hans Knof, Volker Hausen * und Dieter KraffT \\ I. Institut für Experimentalphysik der Universität Hamburg \\ (Z. Naturforsch. 27 a, 162-168 [1972] ; eingegangen am 10. November 1971)
}

\begin{abstract}
An electron attachment ion source was modified in order to be used for quantitative measurement of pure organic substances at pressures up to $1.5 \cdot 10^{-3}$ Torr in the space around the ion source. The ion intensity was determined by counting the electron pulses produced by single ions in the secondary electron multiplier. This resulted in higher sensitivities. The measurements of different organic compounds showed that molecular polymers are held together primarily by hydrogen bonding and by dipole interaction. No molecular polymers could be identified in nonpolar substances. The pressure dependence of the ion intensities indicates that the molecular polymers originate from the gas phase of the reservoir. During the ionization process the hydroxide loses its hydrogen atom as was shown with deuterated samples.
\end{abstract}

In zwei früheren Veröffentlichungen ${ }^{1,2}$ wurde über Untersuchungen an van der Waals-Komplexen organischer Substanzen berichtet. Dabei erfolgte der Nachweis über deren positive und negative Bruchstückionen, sowie mit Hilfe der Elektronenanlagerungsmassenspektrometrie als negative $(\mathrm{nM}-1)$ Komplexionen. Die Ergebnisse an organischen Substanzen können in den allgemeinen Rahmen der Bildung von Kondensationskeimen eingeordnet werden $^{3}$. Die Bildung von Kondensationskeimen wurde bisher in der Massenspektrometrie vor allem an anorganischen Molekülen und an Atomen untersucht.

\section{Apparatur}

Das schon früher beschriebene Massenspektrometer ${ }^{2}$ wurde zur Durchführung der vorliegenden Messungen modifiziert, so daß es auch für höhere Drücke eingesetzt werden konnte. Dadurch gelang es, Massenspektren mit Elektronenanlagerungspeaks zu erhalten, die bei der erreichten stabilen Betriebsweise quantitativ ausgewertet werden konnten.

Neben dem Umbau der Ionenquelle ** wurde vor allem der Ionennachweisteil zur Erhöhung der Nachweisempfindlichkeit völlig neu konstruiert. Statt der bisher verwendeten Integration der elektrischen Impulse am Ausgang des Sekundärelektronenvervielfachers wurden diese mit einem $10 \mathrm{MHz}$-Einkanalmeßplatz gezählt. Dabei wurden die Zählraten bei geringen Intensitäten direkt ausgedruckt oder bei großen Zählraten die verstärkten elektrischen Impulse parallel zum Zähler hinter dem Einkanalmeßplatz integriert und die Spektren mit einem Kompensationsschreiber registriert.

Zur Steigerung der Nachweisempfindlichkeit der Ionen wurde die bisher geerdete Anode des Sekundärelektronenvervielfachers auf etwa $2 \mathrm{kV}$ gelegt und da-

Sonderdruckanforderungen an Dr. H. KNoF, BP-Institut für Forschung und Entwicklung, D-2000 Wedel (Holstein), Moorweg 71. für die vorher auf etwa $-2 \mathrm{kV}$ liegende Kathode geerdet. Dadurch wurden die negativen Ionen nicht durch die negative Kathodenspannung abgebremst und somit mehr Sekundärelektronen ausgelöst. Diese Potentialverschiebung führte $\mathrm{zu}$ einer zehnfachen Intensitätssteigerung.

Die Magnetstromregelung wurde mit dem Zeitgeber des Einkanalmeßplatzes gekoppelt, indem der Zeitgeber parallel zum Zähler Steuerimpulse an eine Schrittmotoreinheit gab, die über ein Netzgerät den Magnetstrom stufenweise steuerten (Abb. 1). Dadurch ließ sich das Magnetfeld in definierten Schritten verändern und während der Zählzeiten konstant halten.

\section{Meßergebnisse}

Die Auswahl der Substanzen war dadurch beschränkt, daß mit dem Massenspektrometer zur Zeit nur Massenspektren bis zur Masse 250 aufgenommen und nur flüssige Proben untersucht werden konnten. Es gelang, eine Reihe charakteristischer organischer Substanzen zu untersuchen, deren Komplexionen in dem vorgegebenen Massenbereich auftreten müßten. Diese Substanzen gehörten zu den folgenden Verbindungstypen:

Alkohole, Aldehyde, Ketone, Fettsäuren, Nitrile $(-\mathrm{C} \equiv \mathrm{N})$, Amine, Nitroverbindungen, Paraffine, Cycloparaffine und Aromate.

Zum Teil wurden auch Spektren mit deuterierten und teildeuterierten Substanzen aufgenommen, um die Komplexpeaks eindeutig zu identifizieren und um die Wasserstoffabspaltung der Molekül- und Komplexionen bei der Ionisierung zu klären.

* Diplomarbeit 1972.

** Patentanmeldung Nr. P 2143 460.6. 


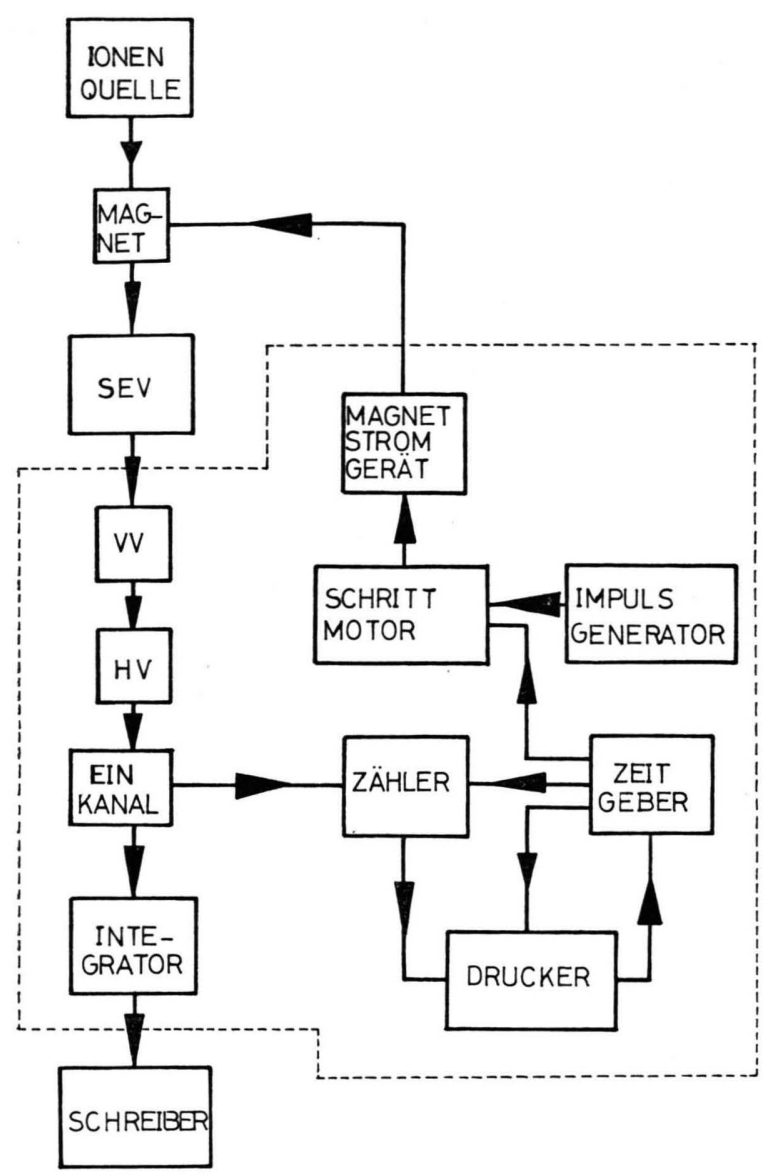

Abb. 1. Magnetstromsteuerung.

\section{Alkohole}

Bei den Alkoholen Methanol, Äthanol und Propanol wurden z. Tl. Komplexe nachgewiesen, die bis zu vier Moleküle enthielten. Die Massenbestimmung konnte durch zusätzliche Messungen deuterierter Substanzen gesichent werden. Damit gelang es auch, den Abspaltungsmechanismus des Protons bei der Bildung der $(n M-1)$-Ionen zu klären.

Sowohl bei Methanol wie auch bei deuteriertem Methanol wurden Komplexe, einschließlich der Trimeren, nachgewiesen (Abb. 2). Die Intensitäten der Monomeren, Dimeren und Trimeren sind in Tab. 1 aufgeführt. Dabei wurden die Intensitäten jeweils auf die schwerste Masse bezogen.

Für Äthanol gelang es außerdem, die Tetrameren nachzuweisen (Abb. 3). Auch hier wurden deuterierte Proben untersucht, die ebenfalls Komplexionen bis zu den Tetrameren lieferten. Die entsprechenden Intensitäten sind in Tab. 1 angegeben.
Schließlich wurde noch Propanol-2 und deuteriertes Propanol-2 untersucht. Auch hier konnten die Komplexe einschließlich der Tetrameren gemessen werden (Abb. 4). Nur beim $d_{8}$-Propanol-2 ließen sich Tetramere wegen ihrer größeren Masse nicht nachweisen. Die Intensitäten sind in Tab. 1 zusammengefaßt.

Ein Vergleich der undeuterierten mit den deuterierten Alkoholen zeigt, daß bei der Ionisation das Wasserstoffatom der Hydroxydgruppe abgespalten wird, und zwar nicht nur bei den Monomeren, sondern auch bei den Polymeren:

$$
\mathrm{R}-\mathrm{CH}_{2} \mathrm{OH}+\mathrm{e} \rightarrow \mathrm{R}-\mathrm{CH}_{2} \mathrm{O}^{-}+\mathrm{H} .
$$

\section{Aldehyde}

Propionaldehyd ist das erste bei Zimmertemperatur und Atmosphärendruck flüssige Element der homologen Reihe der Aldehyde. Es konnten Monomere und dimere Komplexe nachgewiesen werden (Abb. 5), deren Intensitäten in Tab. 2 angegeben sind.

\section{Ketone}

Von den Ketonen wurde Aceton untersucht. Auch die neueren Messungen stimmen mit schon veröffentlichten ${ }^{2}$ überein, d. h. es konnten neben den Monomeren auch die dimeren Komplexe gefunden werden.

\section{Fettsäuren}

Die wegen der Wasserstoffbrückenbindung schon bekannte starke Dimerisation der Fettsäuren konnte massenspektrometrisch bestätigt und darüber hinaus konnten auch die trimeren Molekülkomplexe nachgewiesen werden. Es gelang, wiederum die Massenzahlen durch deuterierte Proben zu bestätigen und mit teildeuterierten Proben die Herkunft des bei der Ionisation abgespaltenen Wasserstoffes aus der Hydroxidgruppe nachzuweisen. Die Reaktionsgleichung für die Ionisation lautet also:

$$
\mathrm{R}-\mathrm{COOH}+\mathrm{e} \rightarrow \mathrm{R}-\mathrm{COO}^{-}+\mathrm{H} .
$$

Bei der Ameisensäure (Abb. 6) wurde neben einer starken Dimerenkonzentration eine schwächere Trimerenkonzentration nachgewiesen (Tab. 3).

Bei der Essigsäure konnten ebenfalls Komplexe bis zur dreifachen Molekülmasse nachgewiesen werden (Abb. 7). In beiden Fällen ist die Intensitätsabnahme zu höheren Komplexen hin wesentlich stärker als bei den Alkoholen (Tab. 3). 
Tab. 1. Intensitätsverhältnisse bei Alkoholen.

\begin{tabular}{llr}
\hline Substanz & \multicolumn{2}{l}{ Massen } \\
\hline $\mathrm{CH}_{3} \mathrm{OH}$ & $31: 63: 95$ & $400: 2: 1$ \\
$\mathrm{CH}_{3} \mathrm{OD}$ & $31: 64: 97$ & $320: 2: 1$ \\
$\mathrm{CD}_{3} \mathrm{OD}$ & $34: 70: 106$ & $510: 3: 1$ \\
$\mathrm{C}_{2} \mathrm{H}_{5} \mathrm{OH}$ & $45: 91: 137: 183$ & $1750: 70: 14: 1$ \\
$\mathrm{C}_{2} \mathrm{H}_{5} \mathrm{OD}$ & $45: 92: 139: 186$ & $13600: 136: 8: 1$ \\
$\mathrm{C}_{2} \mathrm{D}_{5} \mathrm{OD}$ & $50: 102: 154: 206$ & $5000: 200: 25: 1$ \\
$\mathrm{C}_{3} \mathrm{H}_{7} \mathrm{OH}$ & $59: 119: 179: 239$ & $900: 60: 6: 1$ \\
$\mathrm{C}_{3} \mathrm{H}_{7} \mathrm{OD}$ & $59: 120: 181: 242$ & $1960: 280: 20: 1$ \\
$\mathrm{C}_{3} \mathrm{D}_{7} \mathrm{OD}$ & $66: 134: 202: 270$ & $300: 10: 1:-$ \\
\hline
\end{tabular}

Tab. 2. Intensitätsverhältnisse bei Aldehyden.

\begin{tabular}{ccc}
\hline Substanz & Massen & Intensitäten \\
\hline $\mathrm{C}_{2} \mathrm{H}_{5} \mathrm{CHO}$ & $57: 15$ & $16: 1$ \\
\hline
\end{tabular}

Tab. 3. Intensitätsverhältnisse bei Fettsäure.

\begin{tabular}{llr}
\hline Substanz & \multicolumn{1}{l}{ Massen } & \multicolumn{1}{l}{ Intensitäten } \\
\hline $\mathrm{HCOOH}$ & $45: 91: 137$ & $9100: 65: 1$ \\
$\mathrm{HCOOD}$ & $45: 92: 139$ & $1200: 30: 1$ \\
DCOOD & $46: 94: 142$ & $4800: 40: 1$ \\
$\mathrm{CH}_{3} \mathrm{COOH}$ & $59: 119: 179$ & $4500: 30: 1$ \\
$\mathrm{CH}_{3} \mathrm{COOD}$ & $59: 120: 181$ & $6000: 60: 1$ \\
$\mathrm{CD}_{3} \mathrm{COOD}$ & $62: 126: 190$ & $15400: 140: 1$ \\
\hline
\end{tabular}

\section{Stickstoffverbindungen}

Einige organische Stickstoffverbindungen besitzen ein starkes Dipolmoment, ohne zur Wasserstoffbrückenbindung zu neigen.

Bei Nitromethan wurden Komplexe bis zur dreifachen Molekülmasse gefunden (Abb. 8, Tab. 4).

Tab. 4. Intensitätsverhältnises bei Stickstoffverbindungen.

\begin{tabular}{llr}
\hline Substanz & Massen & \multicolumn{1}{c}{ Intensitäten } \\
\hline $\mathrm{CH}_{3} \mathrm{NO}_{2}$ & $60: 121: 182$ & $1050: 7: 1$ \\
$\mathrm{CD}_{3} \mathrm{NO}_{2}$ & $62: 126: 100$ & $550: 5: 1$ \\
$\mathrm{C}_{3} \mathrm{H}_{7} \mathrm{NH}_{2}$ & $58: 117:$ & $60: 1$ \\
$\mathrm{CH}_{3} \mathrm{CN}$ & $40: 81: 122$ & $2100: 30: 1$ \\
$\mathrm{CD}_{3} \mathrm{CN}$ & $42: 86: 130$ & $10800: 180: 1$ \\
$\mathrm{C}_{2} \mathrm{H}_{5} \mathrm{CN}$ & $54: 109: 164$ & $18000: 300: 1$ \\
\hline
\end{tabular}

Die ferner in den Spektren bei $M+46$ und $2 M+46$ auftretenden Linien können evtl. durch Ionen-Molekül-Reaktionen des monomeren bzw. dimeren Moleküls mit dem $\mathrm{NO}_{2}{ }^{-}$-Ion gedeutet werden, da im Spektrum der $\mathrm{NO}_{2}{ }^{-}$-Peak bei der Masse 46 ebenfalls stark hervortritt.

Eine Stickstoffverbindung, die zu einer schwachen Wasserstoffbrückenbindung neigt, ist n-Propylamin.
Hier konnten noch die dimeren Komplexe nachgewiesen werden (Abb. 9). Das gemessene Intensitätsverhältnis der Monomeren zum Dimeren entspricht etwa dem bei den Alkoholen und den Fettsäuren (Tab. 4).

Eine Stoffklasse mit sehr großem Dipolmoment bilden die Nitrile. Hier konnten wieder Komplexe einschließlich der Trimeren nachgewiesen werden.

Bei Acetonitril wurden die Komplexmassen durch Vergleichsmessungen mit deuteriertem Acetonitril bestätigt (Abb. 10). Die Intensitäten sind in Tab. 4 zusammengefaßt.

Entsprechend konnten bei Propionitril Trimere nachgewiesen werden (Abb. 11).

\section{Kohlenwasserstoffe}

Von den unpolaren Kohlenwasserstoffen wurden Benzol, Cyclopentan und Cyclohexan untersucht. Bei diesen Stoffen konnten Komplexionen nicht eindeutig nachgewiesen werden, und das $(M-1)$-Ion trat auch nur bei Benzol deutlich hervor.

\section{Druckabhängigkeit der Ionenintensitäten}

Die Ionenintensitäten der Aceton- und Ameisensäurekomplexe sowie einiger Bruchstückionen in Abbängigkeit vom Druck sind in Abb. 12 eingetragen. Dabei zeigt sich, daß die Steigung der Intensitäten der Molekül- und Komplexionen doppelt so groß ist wie die der Bruchstückionen. Das bestätigt frühere Messungen ${ }^{2}$, bei denen zwischen den Intensitäten der durch Paarbildung in der Druckabhängigkeit erzeugten Bruchstückionen und den durch Elektronenanlagerung gebildeten Molekül- und Komplexionen ebenfalls dieser Faktor gefunden wurde.

\section{Diskussion}

Die untersuchten Substanzen lassen sich in zwei Gruppen einteilen. Eine Gruppe umfaßt die organischen Sauerstoff- und Stickstoffverbindungen, bei denen Komplexe beobachtet werden konnten. Bei den Alkoholen traten Komplexe bis zur vierfachen Molekülmasse auf. Eine andere Gruppe bilden die reinen Kohlenwasserstoffe, bei denen es nicht gelang, Molekülkomplexe nachzuweisen. Es war hier sogar schwierig, Molekülionen zu erhalten. Lediglich bei Benzol und Cyclohexan trat im Bereich des Molgewichtes ein Massenpeak auf, $\operatorname{der} \operatorname{dem}(M-1)$ - 

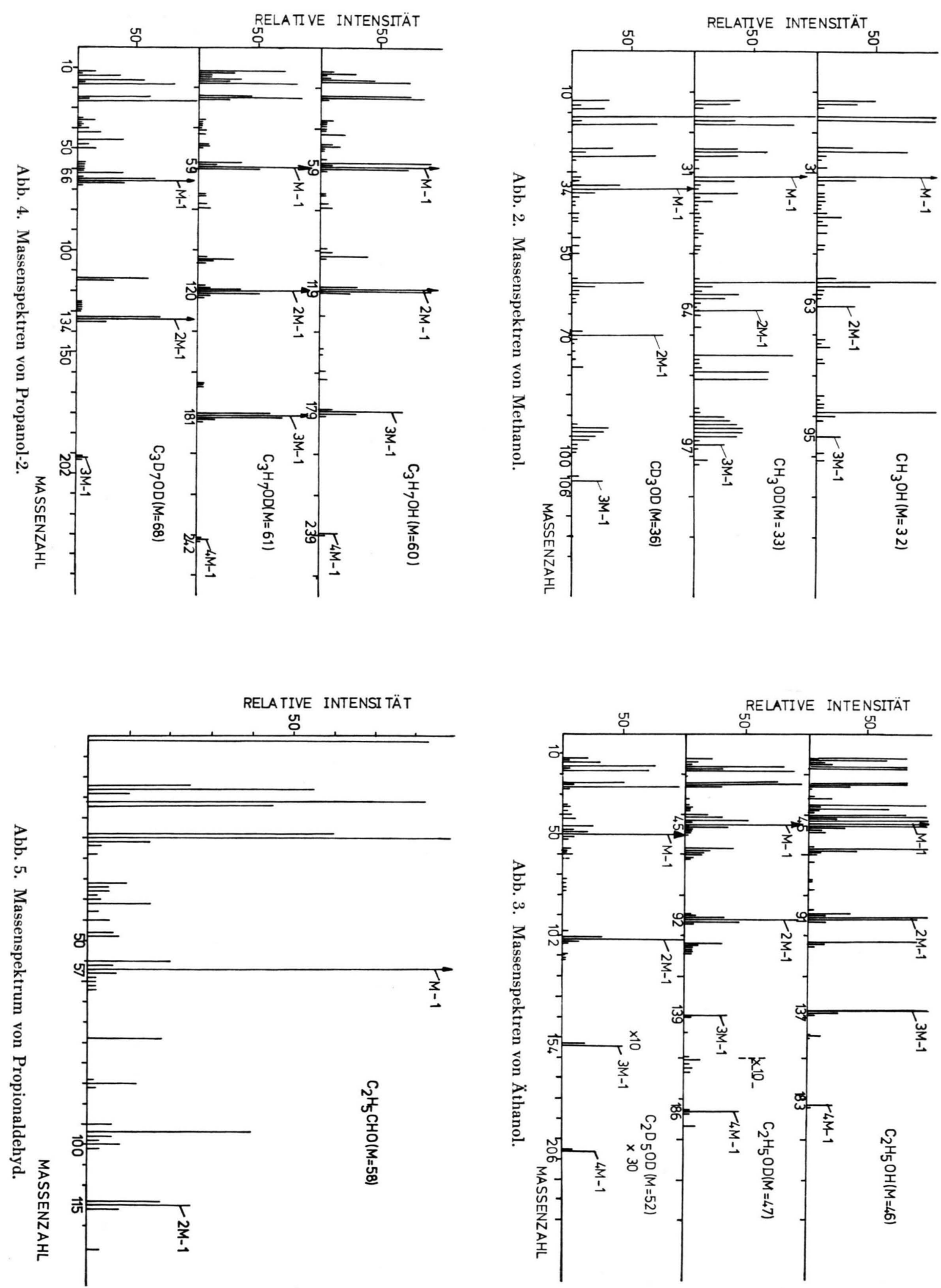


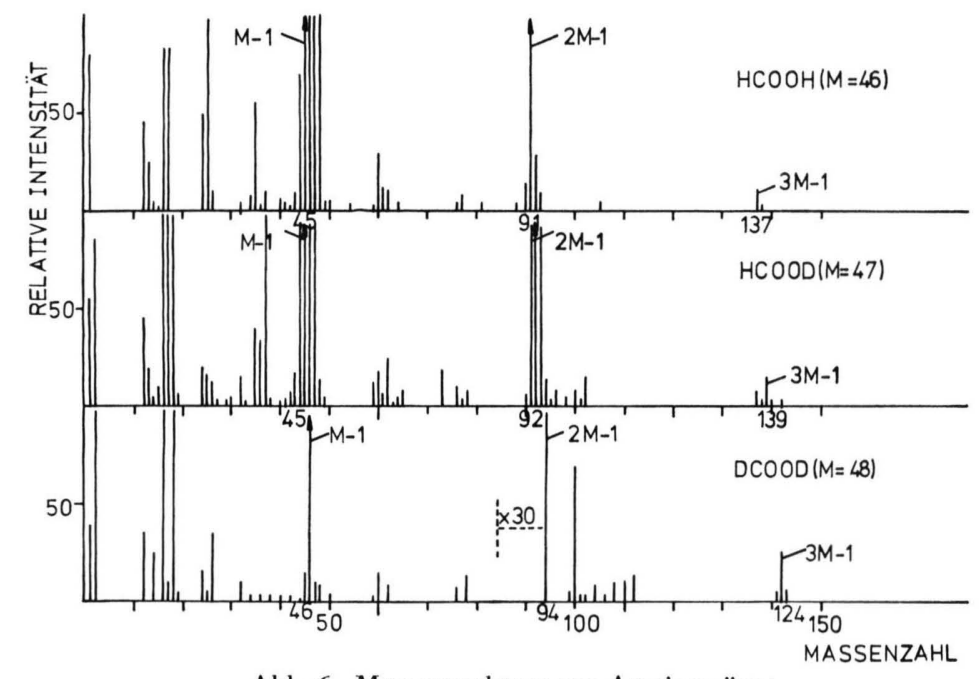

Abb. 6. Massenspektren von Ameisensäure.

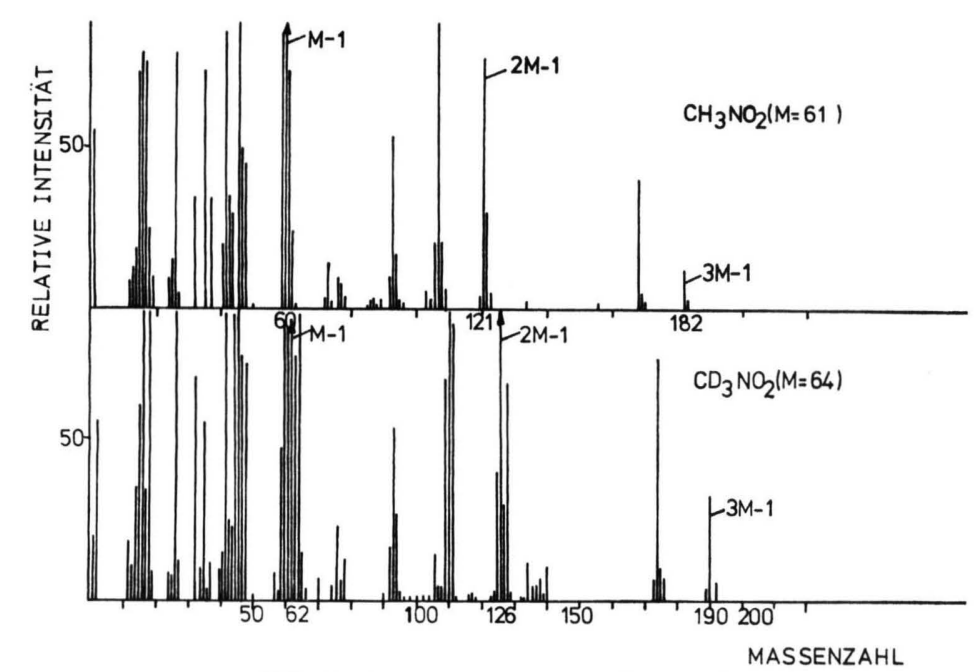

Abb. 8. Massenspektren von Nitromethan.

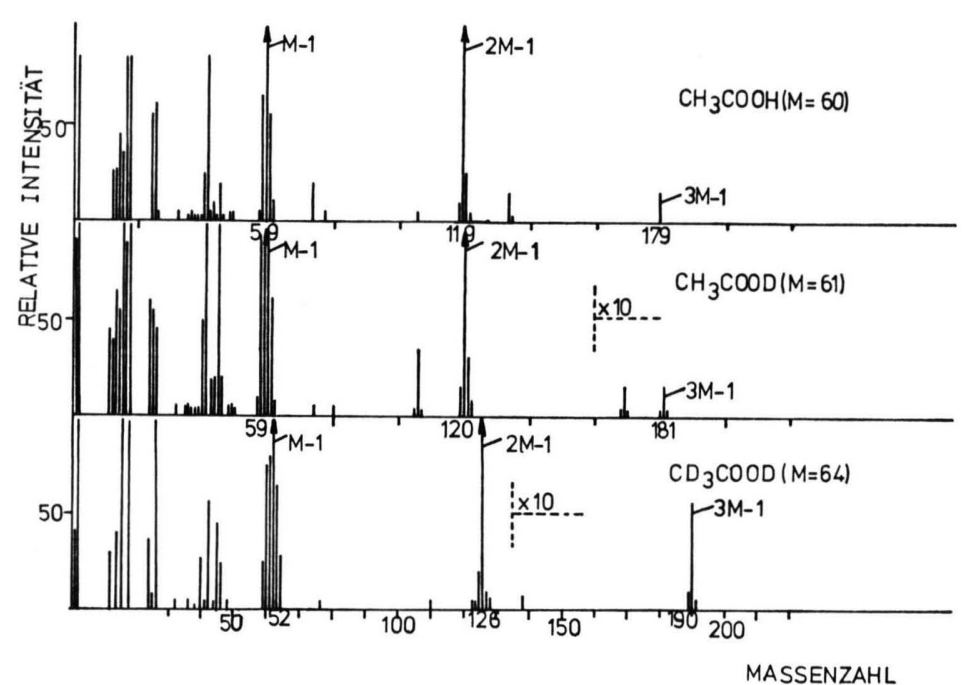

Abb. 7. Massenspektren von Essigsäure.

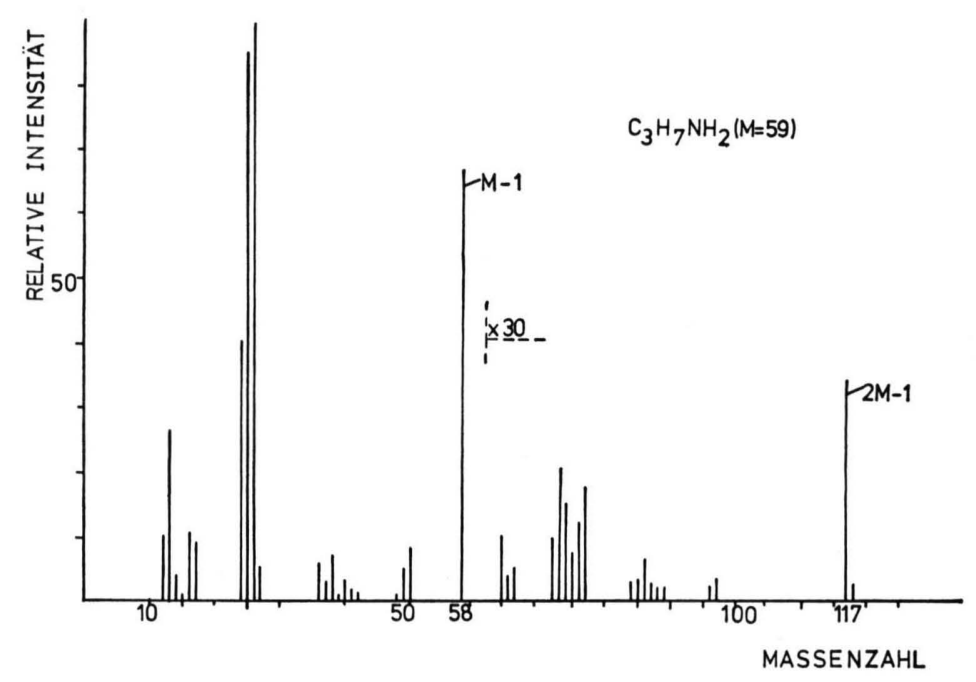

Abb. 9. Massenspektrum von n-Propylamin. 


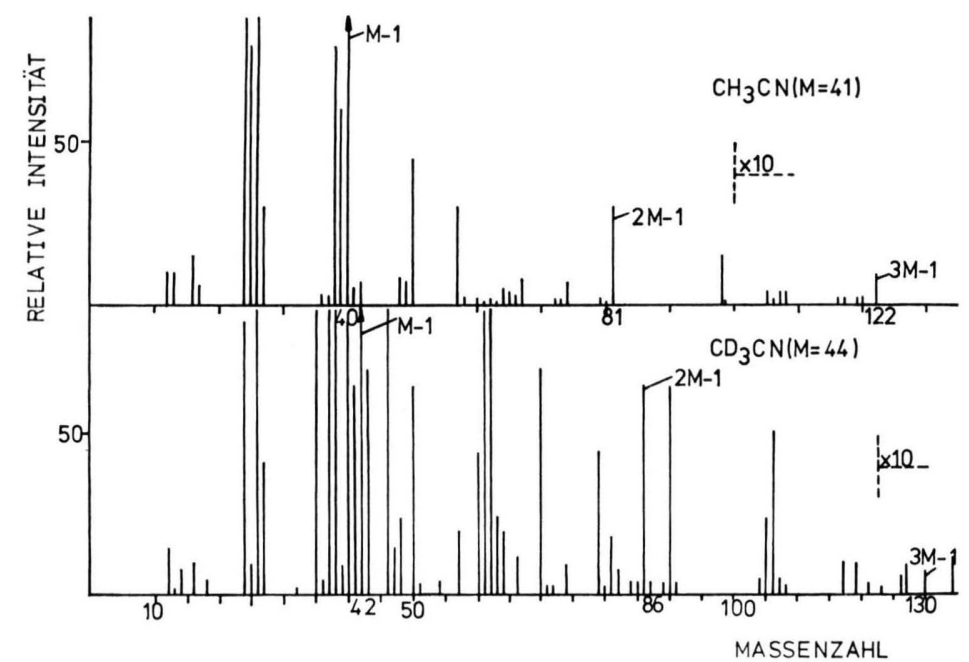

Abb. 10. Massenspektren von Acetonitril.

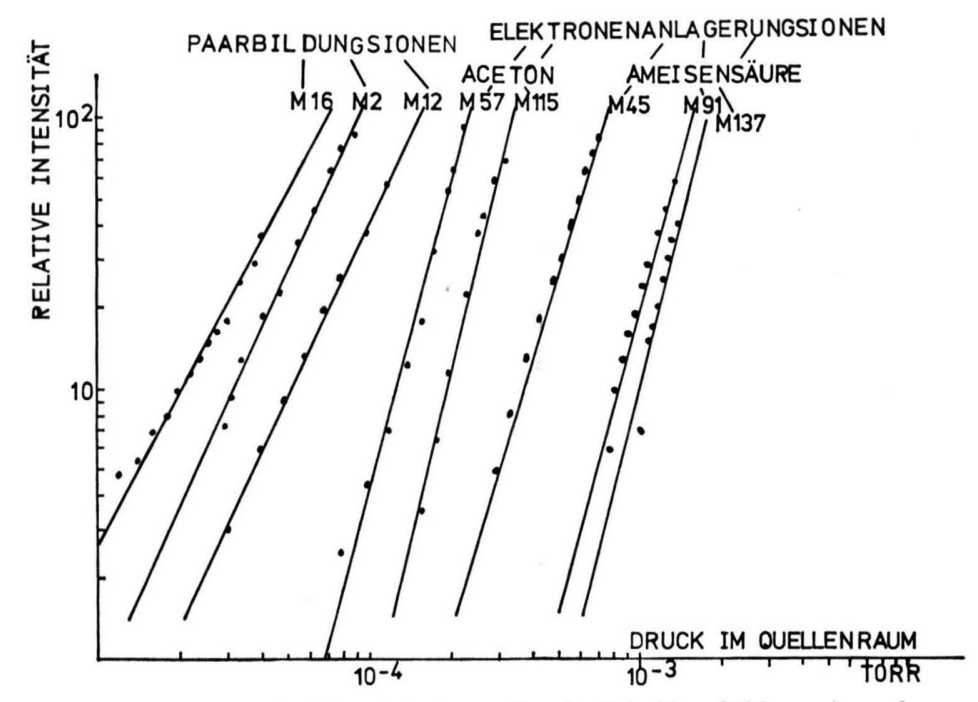

Abb. 12. Druckabhängigkeit von Bruchstück-(Paarbildungs-) - und $(n M-1)$ - (EA) -Ionen.

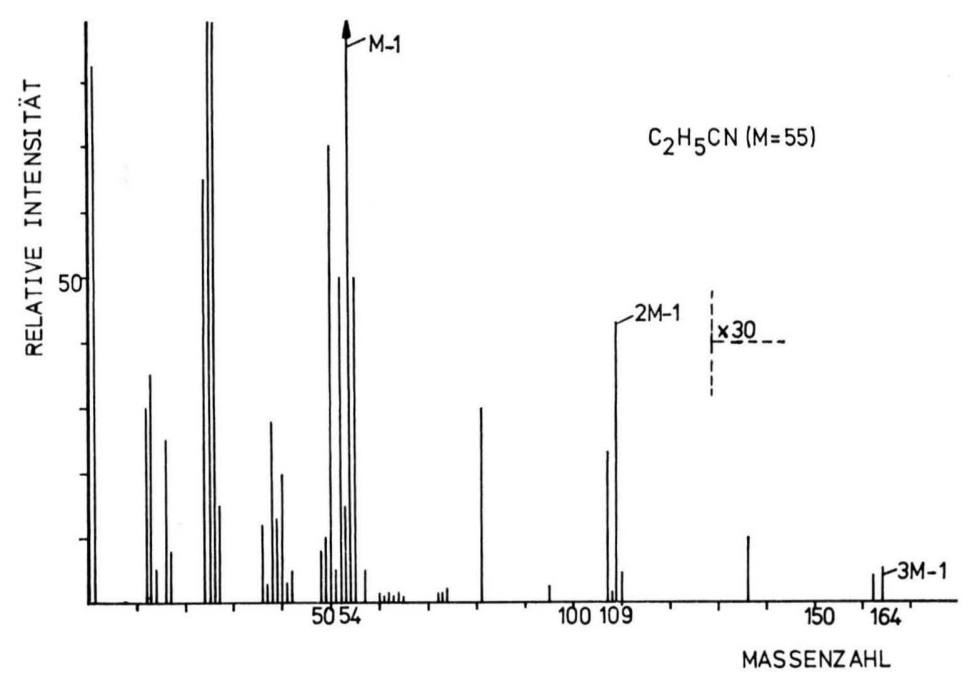

Abb. 11. Massenspektrum von Propionitril.

Tab. 5. Dipolmoment, Polarisierbarkeit und Dampfdruck von Verbindungen.

\begin{tabular}{lllr}
\hline Substanz & $\begin{array}{l}\text { Dipolmoment } \\
\mu \cdot 10^{18} \text { in } \\
\text { el. st. E. }\end{array}$ & $\begin{array}{l}\text { Polarisierbarkeit } \\
\alpha \text { in } \\
\text { el. st. E. }\end{array}$ & $\begin{array}{l}\text { Dampfdruck } \\
\text { in Torr } \\
\text { bei }\left(\vartheta_{z_{i}}\right) \text { in }\end{array}$ \\
\hline $\begin{array}{l}{ }^{\circ} \mathrm{C} \\
\text { Aceton }\end{array}$ & 2,89 & 64 & $245(26)$ \\
Methanol & 1,70 & 37 & $125(26)$ \\
Äthanol & 1,69 & 52 & $59(25)$ \\
Propanol-2 & 1,60 & 41 & $40(24)$ \\
Ameisensäure & 1,52 & & $50(31)$ \\
Essigsäure & 1,74 & 49 & $21(30)$ \\
n-Propylamin & 1,17 & & $400(31)$ \\
Acetonitril & 3,84 & & $100(27)$ \\
Propionitril & 4,05 & & $70(30)$ \\
Nitromethan & 3,44 & 25 & $48(30)$ \\
Propionaldehyd & 2,89 & & $610(29)$ \\
n-Pentan & 0 & 28 & $400(30)$ \\
Cyclopentan & 0 & 26 & $115(28)$ \\
Cyclohexan & 0 & & $118(30)$ \\
Benzol & 0 & &
\end{tabular}


Molekülion der entsprechenden Substanz sicher zugeordnet werden konnte.

Es zeigt sich, daß Substanzen wie Alkohole und Fettsäuren mit einem verhältnismäßig kleinen Dipolmoment, die aber Wasserstoffbrückenbindung aufweisen können, besonders zur Komplexbildung neigen (Tab. 5). Das gleiche Verhalten zeigen auch Substanzen mit starkem Dipolmoment, wie Acetonitril, Propionitril und Nitromethan, obwohl diese Substanzen nicht in der Lage sind, Wasserstoffbrückenbindung zu bilden. Für alle diese Substanzen konnten Komplexe aus drei bzw. vier Molekülen nachgewiesen werden. Dagegen traten bei Substanzen mit kleinem Dipolmoment und schwacher Wasserstoffbrückenbindung, wie n-Propylamin, und bei Substanzen mit mittlerem Dipolmoment, wie Propionaldehyd, nur Komplexe mit der doppelten Molekülmasse im Spektrum auf.

Für die Komplexbildung sind unter den gegebenen Versuchsbedingungen die van der Waalsschen Bindungen zwischen permanenten Dipolen und über Wasserstoffbrücken ausschlaggebend. Bei der Ionisation durch langsame Elektronen wird die überschüssige Stoßenergie durch Abspalten eines Wasserstoffatoms und anschließender Stoßstabilisierung abgegeben, ohne daß die wesentlich schwächere zwischenmolekulare Bindung dabei aufbricht. Das legt folgenden Bildungsmechanismus nahe:

$$
\begin{aligned}
& \mathrm{RH}+\mathrm{e} \rightarrow \mathrm{R}^{*-}+\mathrm{H}, \\
& \mathrm{R}^{*-}+\mathrm{RH} \rightarrow \mathrm{R}^{-}+\mathrm{RH}+E_{\mathrm{kin}} .
\end{aligned}
$$

Bei den Alkoholen und Fettsäuren war es möglich, durch Vergleichsmessungen mit Substanzen verschiedenen Deuterierungsgrades festzustellen, da $\beta$ bei der Ionisierung das Wasserstoffatom der Hydroxidgruppe abgespalten wird.

Die gemessenen Intensitäten der Komplexionen fallen nach höheren Massen hin rasch ab. Dabei sind die angegebenen Werte von der jeweiligen Justierung der Ionenquelle abhängig. Es wurde auf maximale Intensität des schwersten Komplexions justiert. Deshalb ist nur der Vergleich der relativen Intensi-

1 H. Knof u. B. Maiwald, Z. Naturforsch. 23 a, 279 [1968].

2 H. Knof u. D. Krafft, Z. Naturforsch. 25 a, 849 [1970].

3 H. KNOF, Massenspektrometrie von Kondensationskeimen in der Gasphase, Verlag Vieweg, Braunschweig, im Druck. täten zweier verschiedener Substanzen möglich und nicht ein Vergleich ihrer Absolutwerte.

Aus der Druckabhängigkeit der Ionenintensitäten kann auf den Bildungsmechanismus der Ionen geschlossen werden. In Abb. 12 sind Kurven einiger gemessener Druckabhängigkeiten aufgetragen. Dabei treten zwei verschiedene Steigungen auf, die sich um den Faktor 2 unterscheiden. Die Kurven mit der geringeren Steigung, wie für die Bruchstückmassen 12 und 16, können dem direkten Ionisationsprozeß über Paarbildung zugeordnet werden. Deshalb muß die gemessene Steigung, die größer als Eins ist, auf Eins reduziert werden. Der gleiche Reduktionsfaktor geht damit auch in die anderen gemessenen Steigungen ein. Die $(n M-1)$-Ionen von Aceton und Ameisensäure entstehen nach einem anderen Bildungsmechanismus, nämlich durch Resonanzanlagerung niederenergetischer Sekundärelektronen ${ }^{2}$. Da hierbei zwei Schritte, die beide vom Gasdruck abhängig sind, zur Erzeugung der nachgewiesenen Ionen notwendig sind, müssen diese Ionenintensitäten in der Druckabhängigkeit den Faktor 2 gegenüber den direkten Bildungsprozessen aufweisen.

Weiterhin zeigt sich, daß die Druckabhängigkeit der monomeren, dimeren und trimeren Ionen gleich ist. Diese Tatsache läßt sich dadurch erklären, daß die Komplexe schon in der Gasphase im Vorratsbehälter vorhanden sind. Würden diese Komplexe mit dem Dampfdruck im Ionisierungsraum im Gleichgewicht stehen, dann müßten für die verschiedenen Komplexionen auch unterschiedliche Druckabhängigkeiten auftreten.

Wir möchten an dieser Stelle Herrn Prof. NeuerT für sein Interesse und die Möglichkeit, diese Versuche am 1. Institut für Experimentalphysik der Universität Hamburg durchzuführen, unseren Dank aussprechen. Der Deutschen Forschungsgemeinschaft danken wir für die Bewilligung der erforderlichen Sachmittel. H. KNOF erhielt von der Institutsleitung des BP-Institutes für Forschung und Entwicklung die Möglichkeit, diese Arbeiten zu betreuen, wofür er seinen Dank aussprechen möchte.

\footnotetext{
${ }^{4}$ Handbook of Chemistry and Physics, The Chemical Rubber Co., Cleveland 1965.

5 Landolt-Börnstein, Bd. 2, Teil 6, Springer-Verlag, Berlin 1959.
} 